\title{
Dynamic Space-Covered Broadcast Algorithm Based on Neighbor-Degree in Mobile Ad Hoc Wireless Networks
}

\author{
Qiaoqiao $\mathrm{LOU}^{\mathrm{a}, 1}$ and Zhijin $\mathrm{ZHAO}^{\mathrm{b}}$ \\ ${ }^{a}$ School of Telecommunication Engineering of Hangzhou Dianzi University, China \\ ${ }^{\mathrm{b}}$ State Key Lab of Information Control Technology in Communication System of No.36 \\ Research Institute, China Electronic Technology Corporation, China
}

\begin{abstract}
Broadcast is an important link in mobile ad hoc wireless networks (MANETs). In order to improve broadcast coverage, reduce forwarding probability and broadcast collision, a dynamic space-covered broadcast algorithm based on neighbor-degree in MANETs is proposed. The concepts of neighbor-degree, available-angel and available-distance are introduced. The neighbor-degree is used to generate the initial forwarding probability of a node. Based on the available-angle and available-distance, the node weight is proposed to calculate the final forwarding probability, thereby realizing the dynamic selection of forwarding node and reflecting the dynamic space-covered of node. The forwarding strategy is proposed to reduce broadcast collision. Simulation results show that, compared with dynamic probability broadcast and node location based space-covered broadcast, proposed broadcast algorithm reduces the broadcast collision, improves the broadcast coverage, and reduces the forwarding probability when the number of nodes is large.
\end{abstract}

Keywords. MANETs, broadcast coverage, forwarding probability, broadcast collision, forwarding strategy

\section{Introduction}

Broadcast $[1,2]$ is an important link in MANETs (mobile ad hoc wireless networks), which controls the information exchange between nodes. Compared with the broadcast with infrastructure, the broadcast in MANETs is more likely to generate broadcast storm [3] and unreliable broadcast. The specific manifestations are information redundancy, channel contention and signal collision, which seriously affect network performance. Therefore, many scholars have researched the broadcast algorithm [4-6] in MANETs.

Flooding is the simplest approach to broadcast, which ensures a complete coverage but may cause serious redundancy in dense networks, and even broadcast storm. In order to suppress this phenomenon, there are three mainly broadcast algorithms: neighborbased, probability-based [7], and area-based [8]. The simplest broadcast among neighbor-based is self-pruning algorithm [9]. This algorithm selects forwarding nodes based on whether to cover new nodes, regardless of node density, and reduces redundancy generally. Probability-based broadcast algorithm is easy to implement, and

\footnotetext{
${ }^{1}$ Corresponding Author, School of Telecommunication Engineering of Hangzhou Dianzi University, Hangzhou 310018, China; Email:781398337@qq.com
} 
optimizes by compromising overhead and coverage. References $[10,11]$ combined with the self-pruning algorithm to obtain a new dynamic probability broadcast algorithm. The forwarding probability of node is dynamically adjusted with the distance between the sending node and the receiving node, the node density and the transmission range. However, the forwarding strategy of this algorithm results in low broadcast coverage when the number of nodes is small and high redundancy when the number of nodes is large. The area-based broadcasting algorithm considers the additional coverage area of the forwarding node, aiming to reduce redundancy and obtain reliable coverage. References $[12,13]$ proposed a space-covered broadcast algorithm, which divides the coverage of sending nodes into three equal parts, corresponding to three ideal positions. Each area only forwards one node closest to the ideal position in the area. By optimizing the spatial distribution of the forwarding node, the redundancy can be reduced and the broadcast coverage can be ensured, but the broadcast delay is too high. Aiming at the problems of the above algorithms, a dynamic space-covered broadcast algorithm based on neighbor-degree in MANETs is proposed to improve the broadcast coverage when the number of nodes is small and reduce the forwarding probability when the number of nodes is large by optimizing spatial distribution.

\section{Dynamic Space-Covered Broadcast Algorithm Bases on Neighbor-Degree}

Assume that the rectangular coverage area of the network is $10 \times 10 \mathrm{~km}^{2}$, where $\mathrm{N}$ nodes are evenly distributed, and the transmission radius of each node is $r$. In the following, the "sending node" is the node that sends the broadcast packet, which can be divided into "source node" (the first node that sends the broadcast packet) and "non-source node"; the "receiving node" is the node that receives the broadcast packet; the "parent node" is the sending node which receives the broadcast packet for the first time.

We proposed dynamic space-covered broadcast algorithm based on neighbor-degree, which includes forwarding probability calculation and forwarding strategy. Assume that the source node is uniquely determined in each broadcast; each node knows the information of all its two-hop neighbors; each node is equipped with a GPS positioning system, which can obtain its own position information.

\subsection{Ideal Coverage Model}

Assume that the nodes in the network are ideally distributed [13]. Considering each vertex as the sending node, the disk is the transmission range of the sending node. Each sending node is connected to three other sending nodes on the circle. When the sending node is the source node and its coordinate is $\left(x_{1}, y_{1}\right)$, the coordinates of the forwarding nodes on the corresponding circle (denoted as $L s_{-} i, i=1,2,3$ ) [13] are respectively,

$$
L s_{-} 1=\left(x_{1}+r, y_{1}\right), L s_{-} 2=\left(x_{1}-\frac{r}{2}, y_{1}+\frac{\sqrt{3} r}{2}\right), L s_{-} 3=\left(x_{1}-\frac{r}{2}, y_{1}-\frac{\sqrt{3} r}{2}\right)
$$

When the sending node is a non-source node, assuming the coordinate of the parent node is $\left(x_{0}, y_{0}\right)$, the coordinates of the forwarding nodes on the corresponding circle (denoted as $L n s_{-} i, i=1,2$ ) [13] are respectively, 


$$
\left\{\begin{array}{l}
\text { Lns_1 }=\left(\frac{3}{2} x_{1}-\frac{1}{2} x_{0}+\frac{\sqrt{3}}{2} y_{1}-\frac{\sqrt{3}}{2} y_{0}, \frac{3}{2} y_{1}-\frac{1}{2} y_{0}-\frac{\sqrt{3}}{2} x_{1}+\frac{\sqrt{3}}{2} x_{0}\right) \\
\text { Lns_2 }=\left(\frac{3}{2} x_{1}-\frac{1}{2} x_{0}-\frac{\sqrt{3}}{2} y_{1}+\frac{\sqrt{3}}{2} y_{0}, \frac{3}{2} y_{1}-\frac{1}{2} y_{0}+\frac{\sqrt{3}}{2} x_{1}-\frac{\sqrt{3}}{2} x_{0}\right)
\end{array}\right.
$$

Eqs. (1) and (2) represent the ideal node coordinates.

\subsection{Forward Probability Calculation}

Assume that the sending node is $v_{i}$ and the receiving node is $v_{j}$. The neighbor-degree $\delta$ is defined as:

$$
\delta=N\left(v_{j}\right) / \pi r^{2}
$$

Where $N\left(v_{j}\right)$ represents the number of adjacent nodes of the receiving node $v_{j}$.

Using the neighbor-degree $\delta$ instead of the node density of forwarding probability in reference [11], the initial forwarding probability $P_{0}\left(v_{j}\right)$ of the receiving node $v_{j}$ is:

$$
P_{0}\left(v_{j}\right)=1-e^{-\delta r^{2}\left[2 \sin ^{-1}\left(\frac{d\left(v_{i}, v_{j}\right)}{2 r}\right)+\frac{d\left(v_{i}, v_{j}\right)}{2 r^{2}} \sqrt{4 r^{2}-d\left(v_{i}, v_{j}\right)^{2}}\right]}
$$

Where $d\left(v_{i}, v_{j}\right)$ is the distance between the sending node $v_{i}$ and the receiving node $v_{j}$.

Further considering the ideal coverage model, the final forwarding probability $P\left(v_{j}\right)$ can be obtained by combining Eqs. (1) and (2). In the case of the ideal distribution of nodes in the network, the coordinates of forwarding nodes can be calculated according to the ideal coverage model in Section 2.1 and denoted as the ideal node $v_{\text {opt }(l)}$. However, when $N$ is small, there may be a long distance between the ideal node $v_{\text {opt }(l)}$ and the receiving node $v_{j}$, and forwarding node cannot be directly selected from the receiving node $v_{j}$. In order to select the sending node better, the node weight $w_{j}$ is introduced.

Assume that the coordinate of the receiving node $v_{j}$ is $\left(x_{j}, y_{j}\right)$ and the coordinate of the ideal node $v_{\text {opt }(l)}$ is $\left(x_{\text {opt }(l)}, y_{\text {opt }(l)}\right)$. The connection between two nodes is denoted as $L$, so the angle $\gamma_{j l}$ between $L\left(v_{i}, v_{\text {opt }(l)}\right)$ and $L\left(v_{i}, v_{j}\right)$ is:

$$
\gamma_{j l}=\left|\tan ^{-1}\left(\frac{y_{j}-y_{o p t(l)}}{x_{j}-x_{o p t(l)}}\right)\right|
$$

The available-angle of the receiving node $v_{j}$ is defined as $\gamma_{j}=\min \left(\gamma_{j l}\right) \in\left(0,60^{\circ}\right)$. The distance between the receiving node $v_{j}$ and the ideal node $v_{\text {opt }(l)}$ is denoted as $D_{j l}$, and the available-distance of $v_{j}$ is defined as $D_{j}=D_{j c} \in(0, r)$, where $c=\arg \min \left(\gamma_{j l}\right)$. 
The node weight $w_{j}$ is represented by the available-angle $\gamma_{j}$ and the availabledistance $D_{j}$.

$$
w_{j}=\frac{\cos \left(\frac{3}{2} \gamma_{j}\right)}{\sin \left(\frac{\pi D_{j}}{2 r}\right)+1} \in(0,1)
$$

The smaller $\gamma_{j}$ and $D_{j}$, the closer the receiving node $v_{j}$ and the ideal node $v_{\text {opt }(l)}$, the greater the node weight $w_{j}$. Select $v_{j}$ with a large $w_{j}$ as the forwarding node.

Combining $P_{0}\left(v_{j}\right)$ and $w_{j}$, the final forwarding probability $P\left(v_{j}\right)$ of the receiving node $v_{j}$ is:

$$
P\left(v_{j}\right)=\left\{\begin{array}{l}
0, \quad U\left(v_{j}\right)=\varnothing \\
\alpha \cdot P_{0}\left(v_{j}\right)+(1-\alpha) \cdot w_{j}, \quad U\left(v_{j}\right) \neq \varnothing
\end{array}\right.
$$

Where, $U\left(v_{j}\right)$ represents the uncovered neighbors of the receiving node $v_{j}$, set $P\left(v_{j}\right)$ to zero when $U\left(v_{j}\right)=\varnothing ; \alpha \in(0,1)$ is a parameter.

In MANETs, nodes are dynamic, and parameters such as the neighbor-degree $\delta$, the available-angle $\gamma_{j}$ and the available-distance $D_{j}$ will change accordingly. Therefore, $P\left(v_{j}\right)$ also changes dynamically, which can achieve dynamic space-covered of the network.

\subsection{Forwarding Strategy}

When the sending node $v_{i}$ is the source node, three nodes with the largest $P\left(v_{j}\right)$ are selected for forwarding; otherwise, two nodes with the largest $P\left(v_{j}\right)$ are selected for forwarding.

According to $P\left(v_{j}\right)$, set the forwarding delay $T_{j}$ for each forwarding node to alleviate broadcast collision; the larger $P\left(v_{j}\right)$, the smaller $T_{j}$. Assume that the sending node $v_{i}$ needs a time slot to send broadcast packet to the receiving node $v_{j}$. Sort forwarding nodes in descending order according to $P\left(v_{j}\right)$. When $v_{i}$ is the source node, $T_{j}$ is 0,1 and 2 in order; otherwise, it is 0,1 .

\subsection{Broadcast Algorithm}

Based on the ideal node coordinates and two-hop position information, the sending node calculates the forwarding probability $P\left(v_{j}\right)$ of adjacent nodes by Eq. (7) when it first receives a broadcast packet. Then the forwarding strategy is implemented, and the forwarding node and the corresponding forwarding delay are added to the broadcast 
packet. Finally, the receiving node determines whether it is a forwarding node by extracting broadcast packet. If yes, calculate the forwarding probability of adjacent nodes, determine the forwarding node, and forward after the delay expires; otherwise, do not forward.

\section{Algorithm Simulation and Performance Analysis}

Assume that the broadcast radius of each node is $r=3 \mathrm{~km}$. The nodes are considered stationary during broadcast. And each node knows the information of its all two-hop neighbors.

When $\alpha=0.7$ and $N=10,20,30,40,50,60,70,80,90$, the simulation results of proposed broadcast, dynamic probability broadcast [11] and node location based spacecovered broadcast [13] are shown in Figures 1 to 3, respectively.

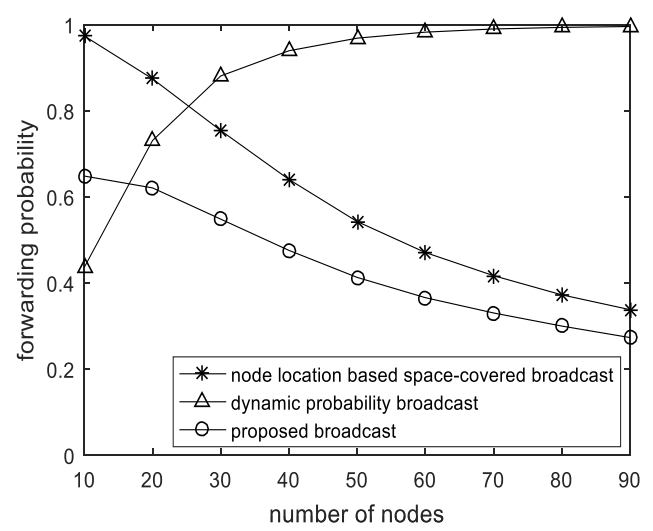

Figure 1. Forwarding probability of different number of nodes

As shown in Figure 1, when $N$ is greater than 20, the forwarding probability of proposed broadcast is lower than dynamic probability broadcast and node location based space-covered broadcast.

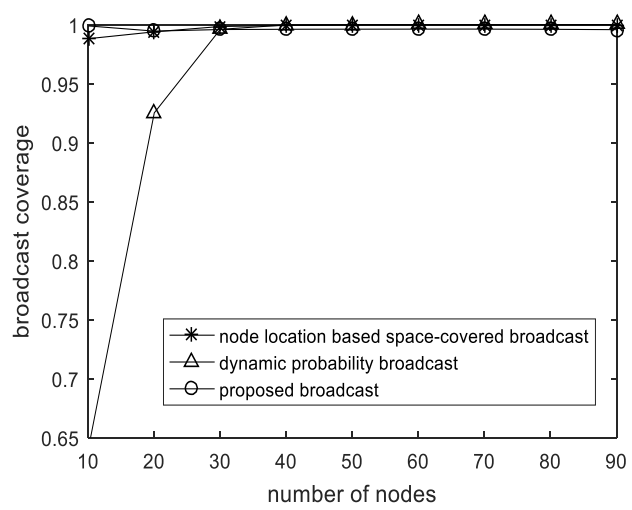

Figure 2. Broadcast coverage of different number of nodes 
As shown in Figure 2, the broadcast coverage of proposed broadcast is almost 1 and has nothing to do with $N$ which is better than dynamic probability broadcast and node location based space-covered broadcast.

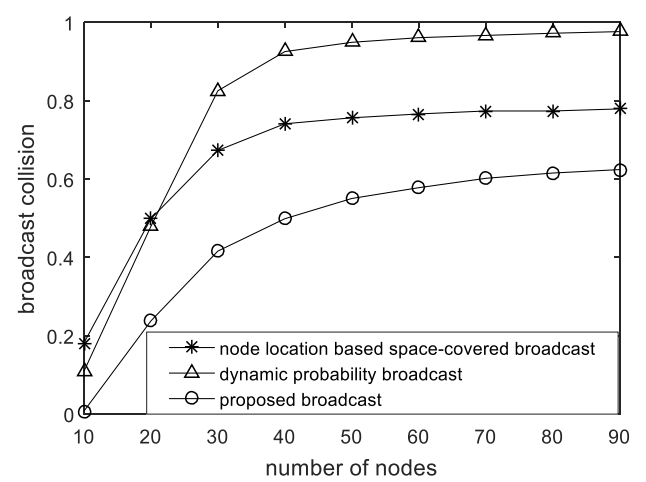

Figure 3. Broadcast collision of different number of nodes

As shown in Figure 3, the broadcast collision of proposed broadcast is the smallest.

\section{Conclusion}

Compared with dynamic probability broadcast and node location based space-covered broadcast, the performance of proposed broadcast is better in forwarding probability, broadcast coverage and broadcast collision.

\section{References}

[1] Y. Ren, A study on broadcasting with low overhead in MANET, Xidian University, 2016.

[2] D. Wang, Y. Song, ECCO: A novel end-to-end congestion control scheme in multi-hop cognitive radio ad hoc networks, IEEE Transactions on Cognitive Communications and Networking 5(1), (2019), 93-102.

[3] B. Yuan, A. Jie, H.B. Zhang, Location aided probabilistic broadcast algorithm for mobile ad-hoc network routing. The Journal of China Universities of Posts and Telecommunications 24(2), (2017), 66-71.

[4] B. Gomathy, D. Sathiya, Scrutiny of broadcasting efficiency with and without clustering in Manet, 2017 International Conference on Innovative Mechanisms for Industry Applications, Bangalore, (2017), 101105.

[5] R. S. R, C. Rajmohan, M. I. H, A modified broadcast algorithm for multi-hop relay MANETs, 2015 Fifth International Conference on Advances in Computing and Communications, Kochi, (2015), 219-222.

[6] A. Fengyang, Research on broadcast algorithms based on wireless network, Qufu Normal University, 2015.

[7] M. B. Yassein, A. Y. Al-Dubai, Inspired counter based broadcasting for dynamic source routing in mobile networks, 2015 International Conference on Computer and Information Technology, (2015), 1455-1459.

[8] B. M. S. Waheed, B. M. Saeed, A. M. Hasan, A. J. Salim, Design of enhanced distance-based neighbor protocol for MANET with the aid of ELECTRE algorithm, 2019 2nd Scientific Conference of Computer Sciences, Baghdad, Iraq, (2019), 57-62.

[9] Y. Saadi, B. Nassereddine, A. Haqiq, CBF evaluation through simulation, 2014 International Conference on Next Generation Networks and Services, Casablanca, (2014), 182-186.

[10] Zohra, F. Tuz, Rahman, Ashikur, Mathematical analysis of self-pruning and a new dynamic probabilistic broadcast for MANETs, 2015 International Conference on Networking Systems and Security, Dhaka, (2015), 1-9. 
[11] R. Rab, A. Rahman, Fatema Tuz Zohra, Analytical modeling of self-pruning and an improved probabilistic broadcast for wireless multihop networks, Ad Hoc Networks 52, (2016), 106-116.

[12] R. Song, J. D. Brown, P. C. Mason, M. Salmanian, H. Tang, HMS: Holistic MPR selection and network connectivity for tactical edge networks, 2015 IEEE Military Communications Conference, Tampa, FL, (2015), 726-731.

[13] L. Q. Bai, Y. Tian, Node location based space-covered broadcast algorithm in ad hoc networks, Journal of Shenyang University (Natural Science Edition) 26(2), (2014), 134-138. 